\title{
Evaluation of QoS Support of AODV and its Multicast Extension for Multimedia over MANETs
}

\author{
Lavanya. Poluboyina \\ Department of ECE, SNIST, Hyderabad-501301, India \\ E-mail: lavanyamam@yahoo.com \\ Sivakumar Reddy. V and Mallikarjuna Prasad. A \\ Department of ECE, MRCET, Hyderabad-500100, India and Department of ECE, JNTUK, Kakinada-533003, India \\ E-mail: vskreddy2003@gmail.com and a_malli65@yahoo.com
}

Received: 14 November 2019; Accepted: 20 December 2019; Published: 08 February 2020

\begin{abstract}
Mobile ad hoc networks (MANETs) have become the prominent set of wireless ad hoc networks with their distinctive characteristics, and ranging several fields of applications. In these networks, the nodes are mobile devices, changing their positions with time, resulting in dynamic topologies of the network. These dynamic environments make the routing of data packets from the source to the destination through multi hoping a difficult task. With the growing popularity of sophisticated mobile devices, the demand for multimedia traffic in such networks is also increasing. Henceforth, to ensure the quality of service (QoS) for multimedia traffic over MANETs has turn into further challenging. This paper focuses on the assessment of built-in QoS support of Ad hoc On-demand Distance Vector (AODV), and its multicast extension MAODV for multimedia traffic (a combination of audio and video) over the mobile ad hoc environment. The work is carried out to measure the achievable QoS metrics like packet delivery ratio, latency, and jitter.
\end{abstract}

Index Terms-AODV, MANET, MAODV, mobile ad hoc network, multimedia, multicasting, ns2, video traffic, VoIP.

\section{INTRODUCTION}

Ad hoc networks are different from infrastructure networks in the way they form the network. They do not rely on any centralized administration or require any routing devices [1]. The nodes themselves are capable of forming a network instantly. In ad hoc networks the nodes, being sophisticated playing dual roles as host and router. Mobile ad hoc networks (MANETs) are one class of wireless ad hoc networks where the nodes are mobile devices changing their positions with time, leading to dynamic topologies of the network. The communication between any two nodes, which are not in direct communication range of each other, happens through multi hoping. MANETs, having distinguished features from other multi hop wireless networks finding applications in various fields e.g., battlefields; emergency situations: where there is a need to have a temporary network without the requirements of any underlying architecture; conferences/convections for exchanging of information by forming an instantaneous network.

A MANET, being a dynamic network posing difficulties for the routing of data packets in the network. In literature, there has been a wide variety of routing protocols and algorithms are proposed, and simulated for various parameters over mobile ad hoc environments. One strategy of classifying the unicast routing protocols based on route finding methodology is: proactive, reactive and hybrid (combines the best features of proactive and reactive) [2]. On-demand protocols provide low convergence times and perform well to proactive type under high mobility environments.

Multicasting [3] is group-oriented communications. It enhances the performance of wireless network by exploiting broadcast nature of the wireless links in delivering multiple copies of datagram to the members of the multicast group that is formed for the objective of sharing information amongst some number of nodes in a network to perform certain special tasks needed in emergency situations, or for collaborative communication. Members of the multicast groups are identified by a unique destination addresses in the network. Multicast routing protocols can be classified broadly as: tree-based and mesh-based. In tree-based, there exists a single path between any pair of nodes in the multicast tree unlike mesh-based where multiple paths may exist between any possible pair of nodes. Tree-based multicast routing protocols offer less routing overhead to mesh-based, making them apt for the dynamic environments of MANETs.

An efficient on-demand routing protocol, Ad hoc Ondemand Distance Vector (AODV) and its multicast extension, Multicast Ad hoc On-demand Distance Vector (MAODV) have been selected for this work because of 
AODV's great suitability to dynamically varying network topologies like MANETs. AODV [4] determines the routes on require whereas MAODV [5], being one of the shared-tree-based and multicast, follows the same AODV's route finding methodologies to establish the multicast routes on demand in the network.

A plethora of research work done on the transmission of constant bit rate (CBR) traffic using the unicast, and multicast routing protocols and a variety of papers have been published so far in the literature, either for audio or video traffic transmission using MANETs for a wide range of routing protocols. In all the cases, the performance of the routing protocols is evaluated taking different sets of evaluation metrics including packet delivery fraction, packets dropped, throughput, delay, delay variation. Real time multimedia applications require quality of service $(\mathrm{QoS})$ parameters like delay and jitter, of small values. On the other hand non-real time applications demand high packet delivery ratio values. In this paper, the performance of AODV is evaluated in terms of a set of performance metrics to check their builtin QoS support for multimedia traffic over the MANETs. And, in parallel the MAODV is also examined for the same defined network and parameters to understand the benefits of multicasting.

This paper is organized into 5 sections. The objective of the paper is discussed in section I. Section II describes related works in the problem area. Section III gives the details of the simulation environment used for performance evaluation of protocols for QoS support. Section IV shows the results, graphs plotted, and the related discussions. Conclusion and future scope of the work are focused in section $\mathrm{V}$.

\section{RELATED WORK}

In [6], the authors have evaluated the performance of two reactive and one proactive routing protocols for realistic video transmission over ad hoc networks using NS-2. Adopted PSNR as the metric and results indicated that DSDV is not suitable for video transmission whereas the AODV is the best compared to DSR.

The authors in [7] simulated (using NS-2) the mobile ad hoc network for MPEG-4 bitstreams using OLSR, AODV and DSR. The overall results have shown AODV is working well with the high load and/or high mobility scenarios.

Chhagan Lal et al. [8] shown that real time multimedia (video) streaming is possible in MANETs however not alone with the traditional routing protocols to supply acceptable QoS. Simulation work has been done using H.264/SVC encoded video sequences with the Qualnet v5.0 for the routing protocols AODV, OLSRv2 and FSR by evaluating EED and PDR.

The performance of AODV, DSR, TORA, OLSR and GRP are investigated in [9] for video streaming applications. The authors have examined for two cases: low resolution video and high resolution video. The simulation work using OPNET simulator revealed that DSR is not fit for video transmission, and video streaming is possible through MANET.

The performance of DSR and AODV is studied for video conferencing in [10]. Audio codec G.723.1 and video codec H.263 were used for simulation and the tool NS-2. Simulation results (E2E delay and packet loss) demonstrated that AODV has covered more area and well performed.

In [11], the performance of two reactive routing protocols is evaluated for GSM quality voice traffic for a MANET. The metrics such as voice E-2-E delay, throughput, voice traffic-sent etc. are evaluated using OPNET Modeler 14.5. Simulation results have shown that for dense MANETs, AODV is the best with GSM voice traffic data.

Gagangeet Singh and Sandeep singh [12] presented a comprehensive simulation study on DSR, AODV, OLSR, TORA, and GRP using OPNET Modeler 14.5 for e-mail and video-conferencing traffic by increasing node density. Network load, throughput, data packets dropped and delay are analyzed for both the traffic and the they have shown that AODV is the best suited one for low node density.

Ref. [13] aims at selecting the best routing protocol out of the available (AODV, DSR and DSDV) for multimedia traffic. A MANET is modeled using ns-2 and the network is analyzed for QoS evaluation metrics. From the graphs plotted, it is concluded that only the reactive protocols are functioning well with the multimedia transmission.

The authors in [14] investigated the impact of network mobility and sparsity on the jitter and PSNR performances of MANETs for video streaming using one reactive and one proactive routing protocols. The overall results demonstrated that AODV is working well than DSDV.

The performance of diverse categories of routing protocols AODV, DSR, OLSR, TORA, and GRP is evaluated in [15] using OPNET modeler simulator for video traffic. The effect of scalability and mobility (considering two scenarios: small scale/low mobility and large scale/high mobility) on the overall performance of the MANET is examined through PDR, end to end delay, throughput (bps) etc. The authors have concluded that the protocols AODV and OLSR are suitable for video traffic.

Khalifa et al. [16] investigated the overall behaviour of the standard MANET routing protocol AODV for video conferencing in the small scale-low mobility scenario using OPNET 17.5 modular.

Most of the efforts in the multimedia transmission over MANETs have mainly utilizing unicast routing protocols. The work in this paper concentrates on multimedia transmission using both the unicast and multicast routing protocols in the mobile ad hoc environment.

\section{SIMULATION WORK ENVIRONMENT}

NS 2.34 [20] is used to simulate the network behaviour considering random waypoint mobility model for node mobility. The network scenario is created in $1000 \times 1000 \mathrm{~m}^{2}$ area with 50 nodes. Network is simulated 
for 10 mins with the node mobility defined for the same duration, i.e., the nodes are in motion during the entire simulation period at pedestrian walking speeds ranging from $0.1 \mathrm{~m} / \mathrm{s}$ to $2.5 \mathrm{~m} / \mathrm{s}$. The MAC layer protocol used is IEEE 802.11 and the propagation model is two-ray ground. Packet size is 512 bytes in CBR and video input cases. In CBR traffic, an acceptable data rate of 4 packets/sec is considered.

The CBR traffic used for evaluation of the protocols is generated using cbrgen.tcl. The standard encoding technique such as ITU-T G.711 codec is used for the generation of audio traffic [17]. Video traffic is generated with Evalvid tool-set [18] taking a 10s video file as input file.

In this paper, first, the performance of AODV is examined for increasing network load i.e., for 10, 20, 30, 40, and 50 connections. Similarly MAODV for 1, 2, 3, 4, and 5 multicast groups with 10 multicast receivers and one multicast sender in each group. QoS support of both the protocols is evaluated for CBR, VoIP (voice over internetworking protocol), and video traffic files separately. Due to dynamic environment of MANETs, for getting consistency in the results, 10 different scenario files are taken and the results are averaged in each case. While defining traffic scenario for AODV, to replicate the MAODV case, the same node was identified as source for 10 receivers and the same strategy followed for 20, 30, 40, and 50 connections.

The second experiment is undertaken protocols are examined for the multimedia by generating two different types of traffic files:

- MM1: 10 CBR connections, 10 VoIP connections, and 10 video connections in case of AODV whereas for MAODV 3 multicast groups, each for one type of traffic.

- MM2: For AODV, only 10 connections covering CBR, VoIP, video traffic and in the case of MAODV one multicast group with three senders initiating three types of traffic transmissions at the same time.

To have single platform for comparison, simulations were run under the same network and traffic scenarios, and initiated the communication at the same times for both the protocols.

\section{RESULTS AND DISCUSSIONS}

The viability of the AODV and MAODV for multimedia traffic is verified quantitatively for a set of QoS parameters like normalized routing load, packet delivery ratio (PDR), latency, and jitter as function of number of connections/multicast groups. PDR expressed in $\%$ is the ratio of a number of data packets received to the transmitted in the network and it is the average value of the network [19]. The ratio of a number of routing packets to the total number of data packets received is taken as the normalized routing load, expressed in \%. Latency is the average value and is the time taken for a packet to reach the destination from the source. Jitter is the variation in delay which is an annoying factor when the real time multimedia transmission is considered. Normalized routing load measures the number of routing packets needed for the successful received data packets.

For MAODV, PDR is defined as the data packets originated at the application layer to the data packets expected to be received by the multicast receivers.

\section{A. PDR}

Fig.s 1 and 2 are showing the plots PDR in $\%$ for AODV and MAODV for different traffic types. It is evident from the fig.s that PDR values decreases with increasing number of either connections, or multicast groups. In this low-mobility network, the reason for fall is overloading of queues at intermediate/forwarding nodes with increase in data packets due to more number of traffic connections. In addition, it is observed that indifferent data packet rates and packet sizes considered for different traffics causing various range of PDR values in each case.

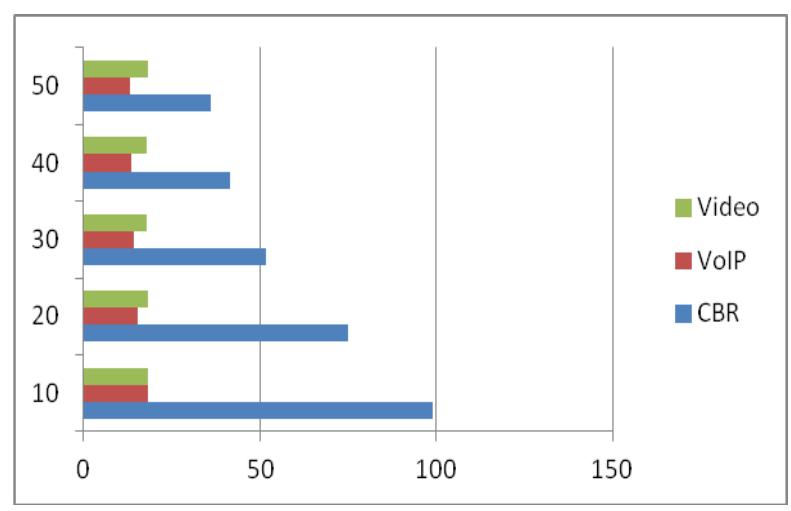

Fig.1. Packet delivery ratio in \% Vs number of connections (AODV)

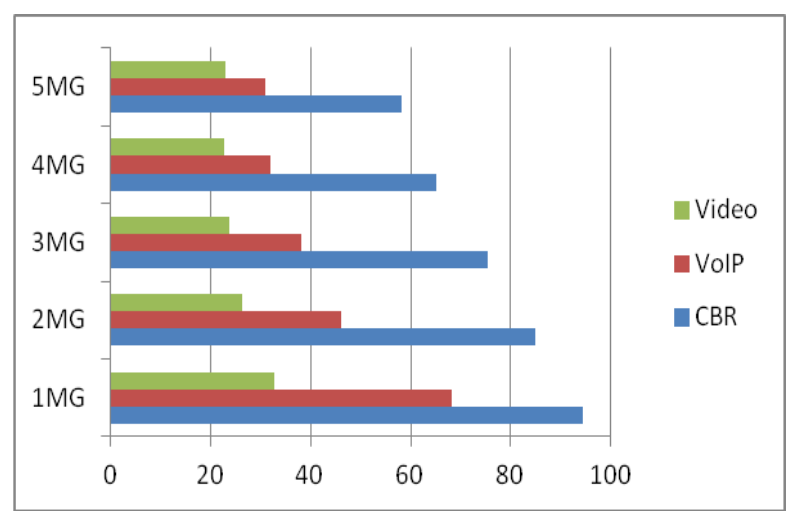

Fig.2. Packet delivery ratio in \% Vs number of multicast groups (MAODV)

The PDR values of AODV are low $(<20 \%)$, and almost identical for VoIP and video traffics. It is noted that the PDR value is not more than $20 \%$ in unicast routing for VoIP and video traffics, whereas in multicast routing it is observed more than $50 \%$ improvement in the PDR values compared to unicast values. Moreover, from fig. 2, it is noted that video PDR fall is much less than the VoIP and CBR cases, and is due to high video packet rates results in low PDR values irrespective of number of traffic 
connections. Though high PDR values with CBR traffic are seen due to its acceptable data rate, only the 10 connections/1MG cases are acceptable having PDR values $>95 \%$.

\section{B. Latency}

Fig.s 3 and 4 representing latency expressed in $\mathrm{ms}$ for AODV and MAODV routing protocols. Small values of latency do not mean good network performance always. As it is calculated for the successful reception of data packets, low latency values could be result of high packet drops. More packet drop rate indicates the situation of network congestion which is the result of high data packets arriving rates into the network which make processors busy and queues overloading. Moreover, it is evident from the graphs with increasing number of traffic connections/multicast groups the latency increases.

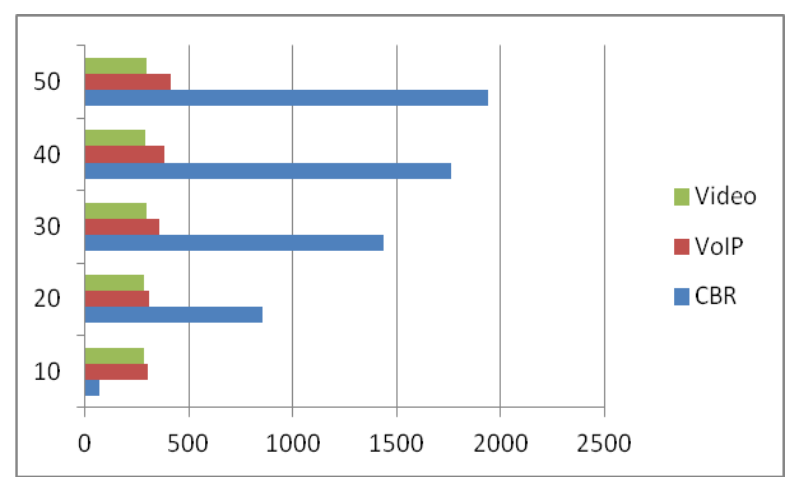

Fig.3. Latency in ms Vs number of connections (AODV)

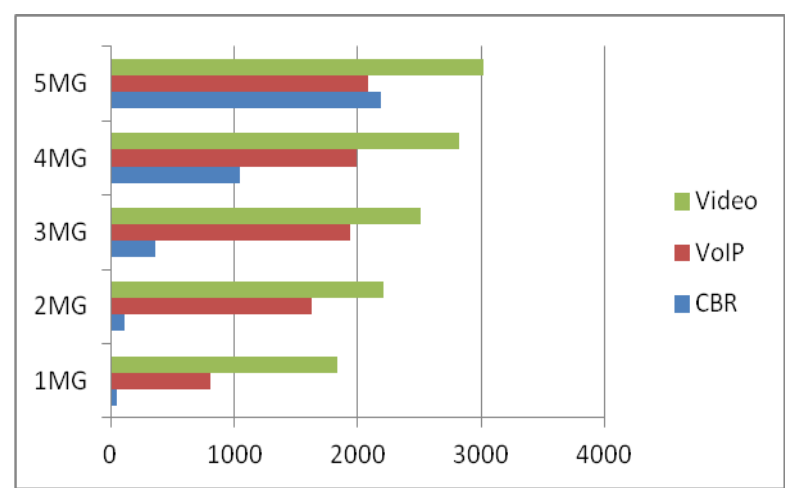

Fig.4. Latency in ms Vs number of multicast groups (MAODV)

Latency must be $<400 \mathrm{~ms}$ for multimedia transmission as per Ref. [21] to provide an acceptable QoS. It is clear from AODV plot, for VoIP and video traffics unlike CBR case, low latency values are identified due to low PDR values that are not acceptable for multimedia transmission. With CBR traffic, acceptable delay values ( $\approx 69 \mathrm{~ms})$ are identified for the case of 10 connections only. In MAODV, high latency values are noted for VoIP and video traffics, in specific, more with video. Moreover, as an enhancement over unicasting, acceptable delay values are identified for CBR traffic with $2 \mathrm{MG}$, and $3 \mathrm{MG}$ cases in addition to $1 \mathrm{MG}$.

\section{Jitter}

Being an important parameter in multimedia transmission, it must be of very small value <1ms [21]. Fig.s 5 and 6 indicate jitter in $\mathrm{ms}$ for AODV and MAODV with CBR, VoIP, and video files as traffic sources. It measures the reliability of the routing protocol over MANETs. AODV plot showing increased values of jitter with increased number of connections, except for video case. As noted, low values of jitter do not indicate the good network conditions; they are result of low PDR values.

In MAODV, increased values are seen with increased number of multicast groups. Much higher values of jitter are perceived with VoIP and video traffics unlike to CBR The minimum value identified with both the protocols irrespective of type of traffic is $\approx 50 \mathrm{~ms}$ which is not an acceptable value for multimedia transmission.

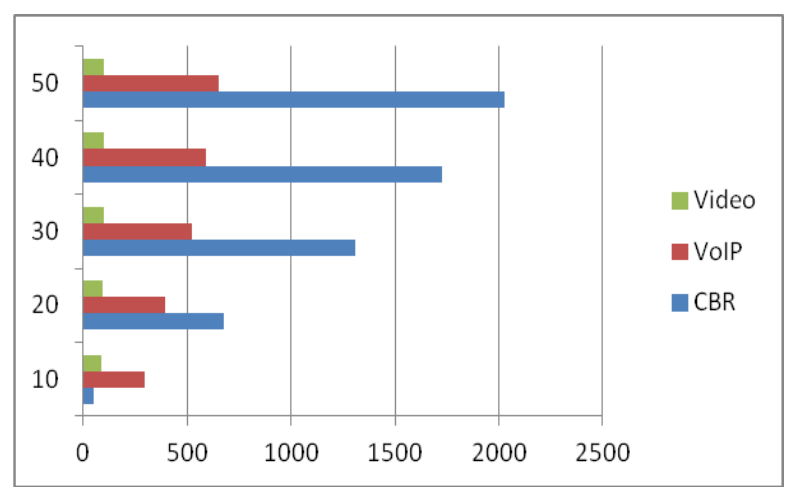

Fig.5. Jitter in ms Vs number of connections (AODV)

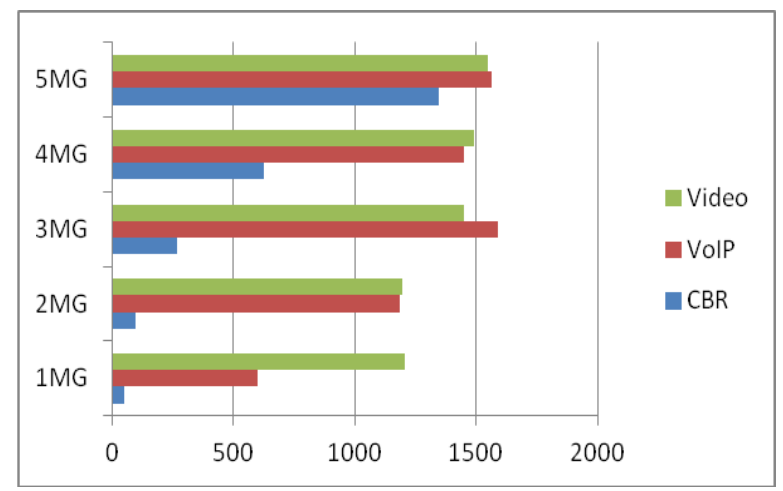

Fig.6. Jitter in ms Vs number of multicast groups (MAODV)

\section{Normalized routing load}

Graphs of normalized routing load expressed in \% for the three traffic types are shown in fig.s 7 and 8 . It is the parameter evaluating the efficiency of the routing protocol in delivering the data packets using as low as possible number of routing packets. A low normalized routing load indicates an efficient routing protocol it is. From the AODV graph, increased values are noticed with increased number of connections except with video traffic With video almost consistent routing loads are observed independent of traffic connections. It is further noticed that routing the VoIP traffic is taking more overhead packets to received packets. Moreover, the amounts of routing loads seen are far much higher with respect to the obtained PDR values irrespective of traffic type. 
The efficiency in routing the data packets to more than one receiver simultaneously is perceived with multicast routing. From the plot of MAODV, it is remarkably noted that the decreasing amount of routing load with increased number of multicast groups. Furthermore, very less amounts of routing loads are identified against the PDR values perceived. For VoIP and video cases, the values are much smaller, in specific, VoIP traffic case almost equal.

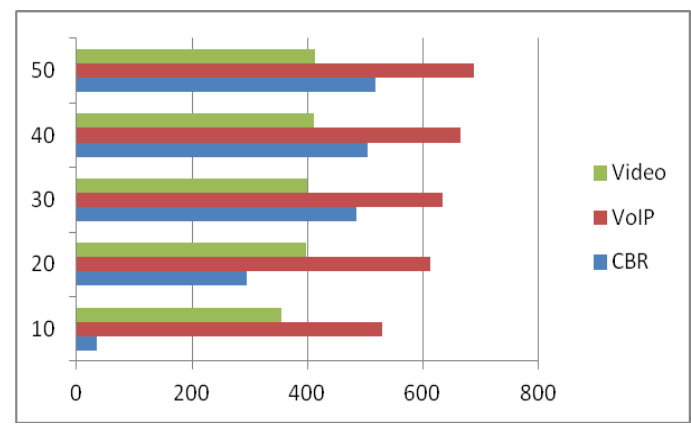

Fig.7. Normalized routing load Vs number of connections (AODV)

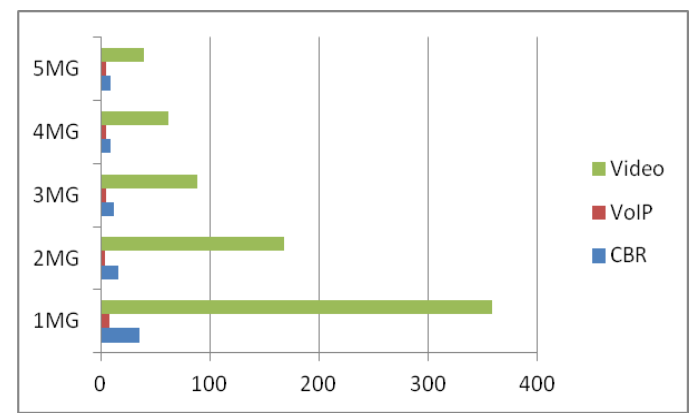

Fig.8. Normalized routing load Vs number of multicast groups (MAODV)

\section{E. For multimedia traffic}

From the sub-sections of A-D it is clearly noted that the performance of both the protocols is acceptable only either for small number of connections or small number of multicast groups, that is, for the defined network for this paper, the acceptable number of connections (multicast groups) is up to 10 (1). Moreover, the benefits of multicasting are clearly visible in the case of normalized routing loads.

The undertaken two protocols are simulated for the defined two multimedia traffic files and the results are illustrated in the fig.s 9-12. It is evident from the PDR plot, though the multicast routing is superior to unicast routing, no protocol is touching atleast $90 \%$ in either case. The continuous transmission of CBR and VoIP packets throughout the simulation making the network congested and led to packet drops. Much higher values of latency are identified with MAODV than AODV in the fig. 10. Acceptable delay values are observed with only AODV, that to in MM1 case which is due to of small PDR values. The jitter graphs clearly showing the protocols unfitness to multimedia traffic.

The resulted PDR values of AODV for MM1 and MM2 are $\approx 22 \%$ whereas the normalized routing loads for
MM1 and MM2 are 404\% and 422\% respectively. For MAODV, the PDR values obtained are 51\% (MM1), $54 \%$ (MM2) and the respective normalized routing loads are $\approx 5 \%$ in both the cases. From this, it is clearly noted that MAODV used very less number of routing packets for the transmission of data packets than AODV, shown in fig. 12. This observation justifies the efficiency of multicast routing protocols. Even though the PDR values of AODV are less than half of the MAODV's, significant differences are found in the normalized routing loads of the two routing protocols.

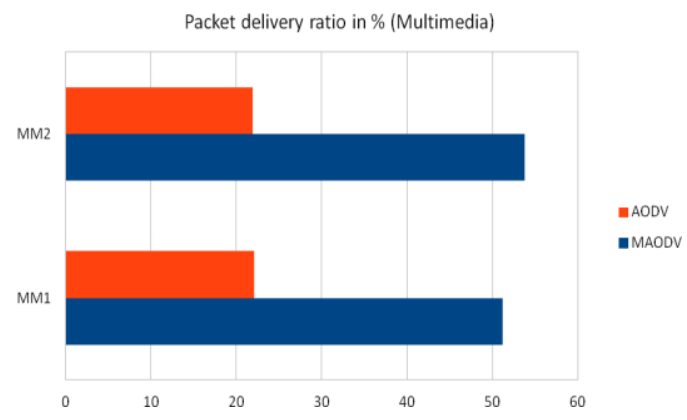

Fig.9. Packet delivery ratio in \% for multimedia

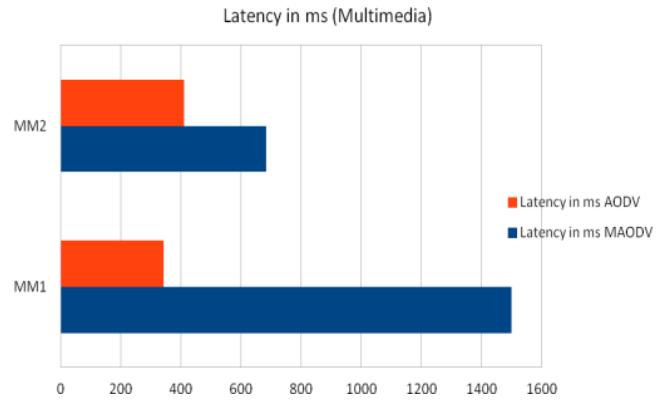

Fig.10. Latency in ms for multimedia

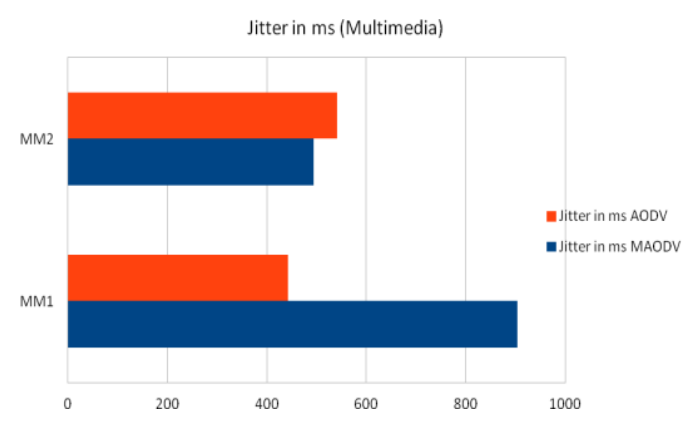

Fig.11. Jitter in ms for multimedia

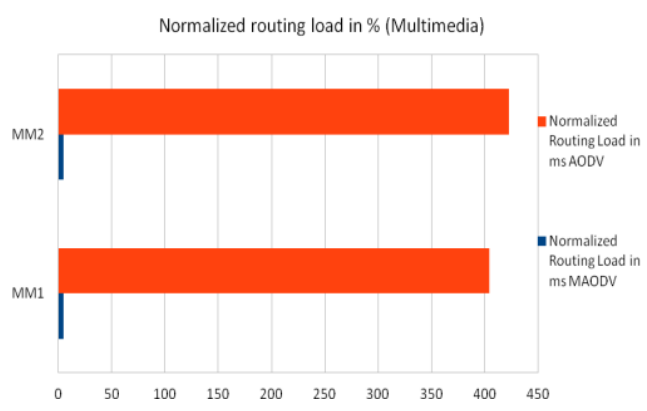

Fig.12. Normalized routing load in \% for multimedia 
From the analysis of the results it is noticed that none of the protocol is suitable for multimedia applications for the considered network environment and parameters.

\section{CONCLUSION}

The performance evaluation and comparison of the familiar and most commonly adapted routing protocol for MANETs, that is, AODV and its multicast extension MAODV for the CBR, VoIP, and video traffics as first experiment. Ten random topologies containing mobile nodes that are moving with random mobilities at pedestrian speeds are created and the results are averaged for consistency. From the obtained results, it is noted that no protocol's performance is acceptable either for VoIP or video traffic irrespective of number of connections/multicast groups.

In the second experiment, the two protocols are examined for the defined two types of multimedia traffic files. Provisioning of acceptable QoS for multimedia applications demand high values of packet delivery ratio, and very low values of latency, and jitter. From the graphs, it is revealed that neither of the protocol's and for none of the case's, the performance is acceptable for supporting multimedia traffic. Only the benefits of multicast routing are clearly observed from the obtained results (plots of PDR, and normalized routing load).

The work done in this paper clearly shown the need for enhancing the two basic protocols to support multimedia traffic. In depth analysis can be done to investigate the reasons for low packet delivery ratio and high values of latency to improve the performance. Further, new techniques can be designed for MAODV (to utilize multicasting) to improve its performance to support the QoS by reducing latency and jitter, over MANETs.

\section{REFERENCES}

[1] Siva Ram Murthy. C, and B. S. Manoj, Ad hoc Wireless Networks: Architectures and Protocols, PHI Pearson Education Inc.: India, 2004, pp.304-307.

[2] Boukerche Azzedine et al., "Routing Protocols in Ad hoc Networks: A Survey," Computer Networks 55, no. 13, pp. 3032-3080, 2011.

[3] Obraczka Katia, and G. Tsuduk, "Multicast Routing Issues in Ad hoc Networks," in IEEE 1998 Int. Conf. on Universal Personal Communications. Conference Proceedings (Cat. No. 98TH8384), vol. 1, pp.751-756, 1998.

[4] Perkins Charles, Elizabeth Belding-Royer, and Samir Das, Ad hoc On-demand Distance Vector (AODV) Routing, no. RFC 3561, 2003.

[5] Royer Elizabeth. M, and Charles E. Perkins, "Multicast Operation of the Ad-hoc On-demand Distance Vector Routing Protocol," in Proceedings of the 5th annual ACM/IEEE Int. Conf. on Mobile computing and Networking, ACM, pp.207-218, 1999.

[6] Ku-Lan Kao, Chih-Heng Ke, and Ce-Kuen Shieh, "Video Transmission Performance Evaluation of Ad hoc Routing
Protocols," In 2006 Int. Conf. on Intelligent Information Hiding and Multimedia, IEEE, pp.181-184, 2006.

[7] Kunagorn Kunavut, and Teerapat Sanguankotchakorn, "Performance Evaluation of Ad hoc Routing Protocols to deliver MPEG-4 Traffic," in 2010 IEEE 12th Int. Conf. on Communication Technology, pp.207-210, 2010.

[8] Chhagan Lal, V. Laxmi, and M. S. Gaur, "Performance Analysis of MANET Routing Protocols for Multimedia Traffic," In 2011 2nd Int. Conf. on Computer and Communication Technology (ICCCT-2011), IEEE, pp.595-600, 2011.

[9] Muhammad Shaffatul Islam, Md. Adnan Riaz, and Mohammed Tarique, "Performance Analysis of the Routing Protocols for Video Streaming over Mobile Ad hoc Networks," Int. J. of Computer Networks \& Communications 4, no. 3, pp. 133-150, May 2012.

[10] Abdullah Md. Ibrahim, M. Muntasir Rahman, A. Ul Ambia, and Zulfiker Mahmud, "Performance of Conferencing over MANET Routing Protocols," ARPN Journal of Systems and Software 2, no. 2222-9833, pp. 214-218, 2012.

[11] Vishal Sharma, Harsukhpreet Singh, Mandip Kaur, and Vijay Banga, "Performance Evaluation of Reactive Routing Protocols in MANET Networks using GSM based Voice Traffic Applications," Optik-Int. J. for Light and Electron Optics 124, no. 15, pp. 2013-2016, 2013.

[12] Gagangeet Singh Aujla, and Sandeep Singh Kang, "Comprehensive Evaluation of AODV, DSR, GRP, OLSR and TORA Routing Protocols with varying number of nodes and traffic applications over MANETs," IOSR $J$. of Computer Engineering 9, no. 3, pp. 54-61, 2013.

[13] Kadam Ajinkya. D, and Sharad S. Wagh, "Evaluating MANET Routing Protocols under Multimedia Traffic," in 2013 Fourth Int. Conf. on Computing, Communications and Networking Technologies (ICCCNT), pp.1-5, 2013.

[14] Sabrina Nefti, and Maamar Sedrati, "PSNR and Jitter Analysis of Routing Protocols for Video Streaming in sparse MANET Networks, using NS2 and the Evalvid Framework," Int. J. of Computer Science and Information Security, vol. 14, no. 3, pp. 1-9, March 2016.

[15] Diaa Eldein Mustafa Ahmed, and Othman O. Khalifa, "Performance Evaluation of Enhanced MANETs Routing Protocols under Video Traffics for Different Mobility and Scalability models using OPNET," American J. of Engineering Research (AJER), 6.7, pp. 329-347, 2017.

[16] Othman O. Khalifa, Diaa Eldin Mustafa Ahmed, Aisha Hassan Abdalla Hashim, and Mudathir Yagoub, "Video Streaming over Ad hoc On-demand Distance Vector Routing Protocol," Bulletin of Electrical Engineering and Informatics 8, no. 3, pp. 863-874, September 2019.

[17] Bouhorma Mohammed, and Anouar A. Boudhir, "VoIP over MANET (VoMAN): QoS \& Performance Analysis of Routing Protocols for Different Audio Codecs," Int. J. of Computer Applications 975, pp.8887, 2011.

[18] V. Sowmya Devi, and Nagaratna P Hegde, "Evaluation and Simulation of Video using Evalvid Tool," IJETT, pp. 198-202, 2017.

[19] S. Corson, and J. Macker, "Mobile Ad hoc Networking (MANET): Routing Protocol Performance Issues and Evaluation Considerations," no. RFC 2501, 1999.

[20] For Network Simulator 2, http://www.isi.edu/nsnam/ns/

[21] https://www.itu.int/rec/T-REC-G.1010-200111-I 


\section{Authors' Profiles}

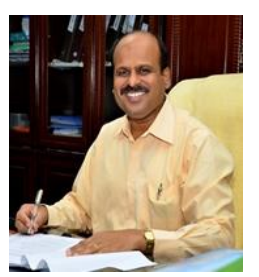

Sivakumar Reddy. V, Alumni of IIT Kharagpur, obtained Ph.D. in the area of Multi-media Signal Processing and Communication Protocols in the year 2008. Received M.Tech. degree in Digital Systems from JNTU College of Engineering, Hyderabad, Telangana and B.Tech. from NBKRIST, Nellore, Sri Venkateswara University, Andhra Pradesh.

$\mathrm{He}$ has an experience of more than 19 years in teaching and industry put together and currently working as Principal, Malla Reddy College of Engineering \& Technology, Hyderabad, Telangana. He published more than 45 research papers in the National and International Conferences and Journals. His areas of interest include Architecture for Signal Processing and Image/Video Coding and Video Communications, Computer Networks, TCP/IP Networks and Protocols, and Multimedia System Design.

Dr Reddy is a fellow of IETE, Life Member of ISTE, and Member of IEEE. Awarded as "Best Teacher" in three consecutive Academic years with citation and cash award. Recipient of "India Jewel Award" for outstanding contribution in the research in the field of Engineering and Technology.

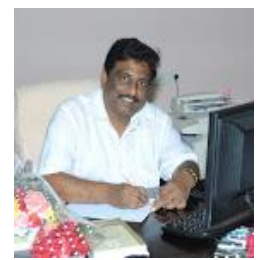

Mallikarjuna Prasad. A received Ph.D. in Electronics and Communication Engineering from University College of Engineering, JNTUH, Hyderabad, Telangana in the year 2009. Completed M.E. from Andhra University, Andhra Pradesh in 1992 and in 1988 done B.Tech.

Andhra Pradesh. from Acharya Nagarjuna University,

He has teaching and industry experience of more than 29 years together and presently working as Professor in the department of Electronics and Communications Engineering, JNTUK, Kakinada, Andhra Pradesh, India. He is having more than 50 research publications in the National and International Conferences and Journals. His research interests include Bio Medical Signal/Image Processing, Wireless Communications, Antennas, VLSI, and Electronic Instrumentation.

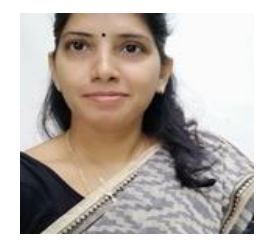

Lavanya. Poluboyina completed B.Tech. in Electronics and Communication Engineering vfrom Vignan's Engineering College, Jawaharlal Nehru Technological University, Hyderabad, Andhra Pradesh in 2004 and done M.Tech. in Digital Systems and Computer Engineering from University College of Engineering, JNTUH, Hyderabad, Telangana in the year of 2009 .

She has teaching experience of more than 15 years and presently working as Assistant Professor in the department of Electronics and Communication Engineering, Sreenidhi Institute of Science and Technology, Hyderabad, Telangana. Her areas of interest are Advanced Data Communications, Wireless Communications and Networks, Routing in Wireless Networks, Wireless Ad hoc Networks, and Mobile Ad hoc Networks. She is a Member of IEEE.
How to cite this paper: Lavanya. Poluboyina, Sivakumar Reddy. V, Mallikarjuna Prasad. A, "Evaluation of QoS Support of AODV and its Multicast Extension for Multimedia over MANETs", International Journal of Computer Network and Information Security(IJCNIS), Vol.12, No.1, pp.13-19, 2020. DOI: $10.5815 /$ ijcnis.2020.01.02 